\title{
Optimization of the Global Re-entry Vulnerability Index to Minimise Cycle Length Dependency and Prediction of Ventricular Arrhythmias during Human Epicardial Sock Mapping
}

\author{
Michele Orini $^{1,2}$, Peter Taggart ${ }^{1}$, Martin Hayward $^{3}$, Pier D Lambiase ${ }^{1}$ \\ ${ }^{1}$ University College London, London, United Kingdom \\ ${ }^{2}$ Barts Heart Centre, St Bartholomew's Hospital, London, United Kingdom \\ ${ }^{3}$ University College London Hospitals, London, United Kingdom
}

\begin{abstract}
The re-entry vulnerability index $(R V I)$ is an activationrepolarization marker recently proposed to predict sites of ventricular tachycardia (VT) initiation. RVI is inversely related to the probability of establishing a re-entry. The aim of this study was to characterize the CL dependency of RVI, assess different methods for CL-dependency corrections and test the capability of RVI to predict ventricular arrhythmias. Twenty-four subjects underwent whole heart epicardial mapping using a multi-electrode sock enabling the recording of 240 unipolar electrograms. Ventricular pacing was delivered at CLs decreasing from 600 to $350 \mathrm{~ms}$ in steps of $50 \mathrm{~ms}$. In a separate study, 1 patient went into VT during steady state pacing. Predisposition to $V T$ was assessed by using the 10th percentile RVI, termed global RVI. The results show that own to CL dependency of local repolarization, there was a strong positive association between RVI and CL. Local repolarization detrending and correction with the Bazett's formula eliminated the CL dependency, while a weak association was found after correction with the Fredericia's formula. In the patient who developed VT, global RVI was significantly lower than in the patients who did not develop VT. Corrections for $C L$ dependency enhanced these differences. In conclusion, detrending and Bazett's corrections effectively compensated for the CL dependency of RVI and global RVI may reveal predisposition to ventricular arrhythmias. Further analysis is necessary to establish the role of RVI for risk stratification.
\end{abstract}

\section{Introduction}

The re-entry vulnerability index (RVI) is an activationrepolarization marker recently proposed to predict sites of reentrant ventricular tachycardia (VT) initiation [1,2]. RVI combines information from proximal electrical repolarization and distal activation to assess the likelihood of estab- lishing the necessary conditions for a re-entrant circuit. It is defined as the interval between repolarization time (RT) at a site proximal to a line of functional conduction block and activation time (AT) at an adjacent site [1]. A negative RVI indicates that repolarization at a given site is faster than depolarization at an adjacent site and therefore that wave-front propagation is possible along the axis between the two sites. The lower the RVI, the more vulnerable the tissue. The primary scope of RVI is to predict sites vulnerable to re-entry and guide catheter ablation therapy without the need of inducing and mapping VT, which for many patients may be unfeasible and dangerous. However, since low RVI is mechanistically related to re-entry, the lowest RVI may also be used to assess patients susceptibility to reentrant arrhythmia. In this respect, cycle length dependency of repolarization $[3,4]$ may constitute a limitation as it can bias RVI estimates, which can change due to heart rate rather than cardiac risk. This paper investigates corrections for RVI heart rate dependency. The aims of the study are to (1) Characterize the interaction between RVI and other important electrophysiological indices, including CL, in the intact human heart; (2) Propose and asses corrections to reduce RVI cycle length dependency; (3) Provide a preliminary assessment of RVIs ability to predict VT.

\section{Material}

During cardio-thoracic surgery, 25 subjects underwent whole heart epicardial mapping using a multi-electrode sock enabling the recording of 240 unipolar electrograms $[3,5]$. Unipolar electrograms were recorded at a sampling frequency of $2 \mathrm{KHz}$, band-pass filtered beteen $0.05-500$ $\mathrm{Hz}$, and referenced against the rib retractor. Drive trains of ventricular pacing were delivered at cycle lengths (CL) decreasing from $600 \mathrm{~ms}$ to $350 \mathrm{~ms}$ in steps of $50 \mathrm{~ms}$. In one patient, ventricular pacing at CL $=650 \mathrm{~ms}$ was maintained for about $90 \mathrm{~s}$ and discontinued afterwards because 
of hemodynamic instability and ST changes appearing on electrocardiogram. After 3 spontaneous sinus beats the rhythm became unstable with short runs of VT that quickly degenerated into fibrillation [6]. The study was stopped and the normal surgical intervention continued successfully after cardioversion.

\section{Methods}

\subsection{Electrophysiological measurements}

Data were exported and analyzed off line with bespoke software as in previous studies $[3,7,8]$. After removing the pacing artifacts and band-pass filtering between $0.5-20 \mathrm{~Hz}$, local AT and RT were measured as the time of the maximum negative downslope during the QRS complex and maximum positive upslope of during the Twave, respectively [9]. The activation-recovery interval, $A R I=R T-A T$, was used as standard surrogate for the local action potential duration. For each drive train, the median AT, RT and ARI were calculated and used to estimate RVI and other electrophysiological indices at the six different CLs.

The RVI at each recording location $i, R V I_{i}$, was calculated taking the minimum difference between RT at site $i$, and AT at neighboring sites $j$ comprised within a $20 \mathrm{~mm}$ radius [1]:

$$
R V I_{i}=\min _{j}\left(R T_{i}-A T_{j}\right)
$$

In this expression, the minimum value is taken to ensure high sensitivity to vulnerable sites. Local dispersion of activation, $D_{\mathrm{AT}}$, and repolarization, $D_{\mathrm{RT}}$, were estimated as the range between the 10th and 90th percentile of AT and RT within the same radius, respectively. For each patient, the global RVI, $R V I_{\mathrm{G}}$, was measured as the 10th percentile of the $R V I_{i}$ distribution, which represents a robust estimate of the global minimum RVI.

\subsection{Corrections for CL dependency}

For each patient and cycle length, RVI corrected for CL dependency was estimated by modifying RT prior to RVI calculation as follows:

1. Detrended global RVI, $R V I_{\mathrm{G}, \mathrm{D}}$ : The median RT was subtracted to all RT. This correction is based on the assumption that CL dependency of repolarization is spatially homogeneous and does not affect the global dispersion of repolarization.

2. Bazett's correction, $R V I_{\mathrm{G}, \mathrm{B}}$ : Local RTs were corrected using the Bazett's formula, $R T_{c}=R T / \sqrt{C L}$.

3. Fredericia's correction, $R V I_{\mathrm{G}, \mathrm{F}}$ : Local RTs were corrected using the Fredericia's formula, $R T_{c}=R T / \sqrt[3]{C L}$.
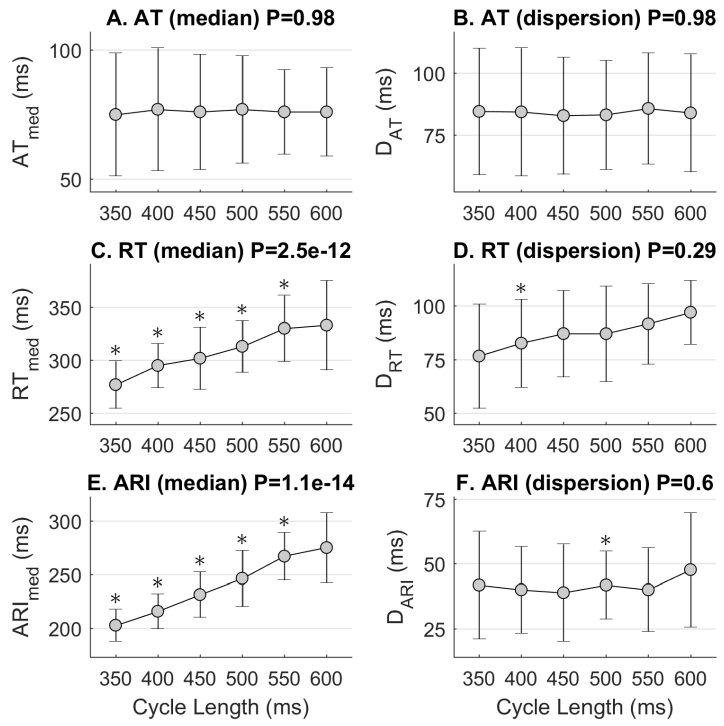

Figure 1. Cycle length dependency of intra-patient median AT, RT and ARI (panels on the left) and global dispersion of AT, RT and ARI (panels on the right). Circles and bars represent median and adjusted median absolute deviation. Asterisks represent pair-wise significant differences with respect to distributions for $C L=600 \mathrm{~ms}$. P-values obtained though group-wise test are given on top of each panel.

\subsection{Statistical Analysis}

Linear correlation was measured with the Spearman's correlation coefficient. Central moment and variability were measured with median and adjusted median absolute deviation, estimated as the median absolute deviation multiplied by 1.483 to approximate the standard deviation. The Wilcoxon signed-rank test was used for pairwise comparisons, while the Kruskal-Wallis test was used for group-wise comparisons. $P<0.05$ was considered significant.

\section{4. $\quad$ Results}

After removing recordings with $\mathrm{SNR}<12 \mathrm{~dB}, 21,748$ RVI estimates were obtained pooling together estimates from all non-VT patients and CLs. Median AT and global AT dispersion were not associated with CL (Fig. 1A-B). Median RT significantly decreased with CL, from $333 \pm 42$ $\mathrm{ms}$ for $C L=600 \mathrm{~ms}$ to $277 \pm 22 \mathrm{~ms}$ for $C L=350$ ms $(P<<0.05$ for both group-wise and pair-wise, Fig. 1C), while global dispersion of RT only showed a non-significant trend to decrease with CL $(P=0.29$ for group-wise comparisons, Fig. 1D). This result suggests that RT detrending may compensate for the CL dependency of global RVI. Median ARI and global dispersion of 
Table 1. Spearman's correlation coefficients between local $R V I_{i}$ and local electrophysiological parameters. Results are reported as median \pm adjusted median absolute deviation.

\begin{tabular}{c|cccccc}
\hline \hline CL & $350 \mathrm{~ms}$ & $400 \mathrm{~ms}$ & $450 \mathrm{~ms}$ & $500 \mathrm{~ms}$ & $550 \mathrm{~ms}$ & $600 \mathrm{~ms}$ \\
\hline AT & $0.13 \pm 0.24$ & $0.25 \pm 0.23$ & $0.22 \pm 0.26$ & $0.18 \pm 0.2$ & $0.18 \pm 0.19$ & $0.14 \pm 0.29$ \\
ARI & $0.68 \pm 0.14$ & $0.71 \pm 0.16$ & $0.65 \pm 0.11$ & $0.7 \pm 0.092$ & $0.68 \pm 0.12$ & $0.74 \pm 0.1$ \\
RT & $0.58 \pm 0.19$ & $0.58 \pm 0.15$ & $0.56 \pm 0.17$ & $0.59 \pm 0.21$ & $0.62 \pm 0.2$ & $0.57 \pm 0.27$ \\
$D_{\text {AT }}$ & $-0.45 \pm 0.073$ & $-0.44 \pm 0.083$ & $-0.52 \pm 0.1$ & $-0.5 \pm 0.11$ & $-0.5 \pm 0.078$ & $-0.51 \pm 0.095$ \\
$D_{\text {ARI }}$ & $-0.31 \pm 0.13$ & $-0.39 \pm 0.12$ & $-0.38 \pm 0.15$ & $-0.37 \pm 0.1$ & $-0.4 \pm 0.13$ & $-0.29 \pm 0.21$ \\
$D_{\text {RT }}$ & $-0.13 \pm 0.13$ & $-0.17 \pm 0.086$ & $-0.2 \pm 0.087$ & $-0.25 \pm 0.13$ & $-0.23 \pm 0.079$ & $-0.24 \pm 0.15$ \\
\hline \hline
\end{tabular}
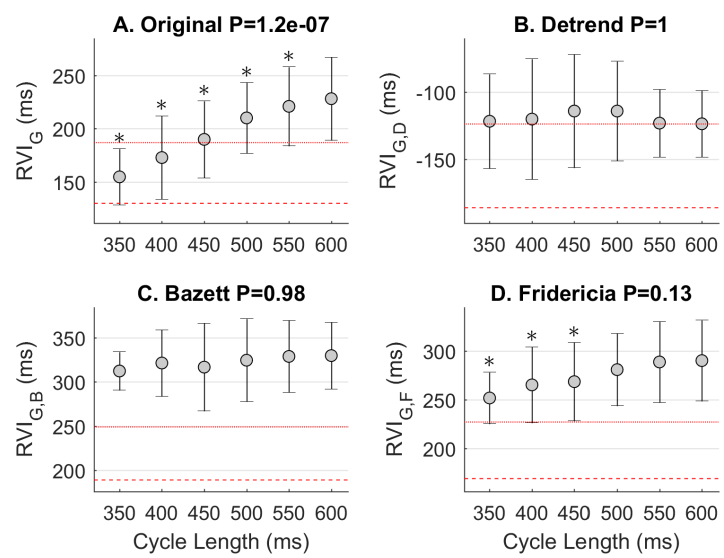

Figure 2. Cycle length dependency of global RVIs before and after CL correction. Circles and bars represent median and adjusted median absolute deviation estimated among patients without VT. Dotted and dashed horizontal lines represent the global RVI of the patient who developed VT, at the onset of pacing and about $90 \mathrm{~s}$ later before VT onset, respectively. Asterisks represent pair-wise significant differences with respect to distributions for $C L=600 \mathrm{~ms}$.

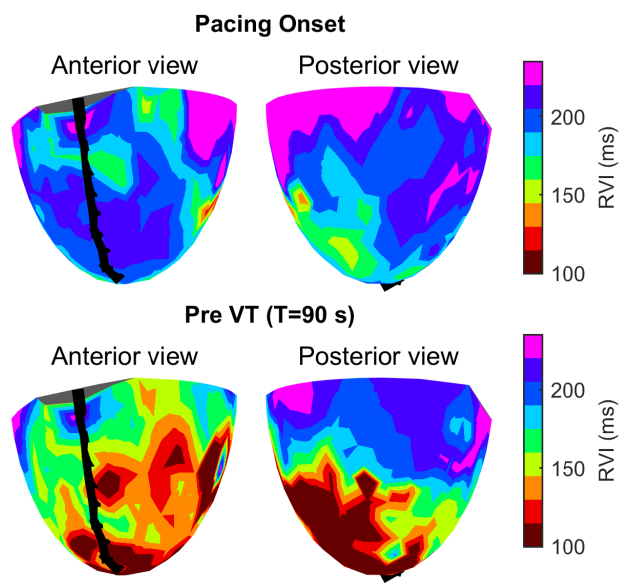

Figure 3. RVI map in the one patient who developed VT at the onset of pacing (top panels) and about $90 \mathrm{~s}$ later, before VT onset (bottom panels). The solid black line represents the LAD.
ARI presented similar association with $\mathrm{CL}$ as RT ( Fig. 1EF).

Local RVI, $R V I_{i}$, showed a moderate to strong correlation with local ARI (about 0.7), RT (about 0.6) and local dispersion of AT (about -0.5) for all CLs (Table 1). Global RVI was strongly associated with CL (fig. 2A). The one patient that went into VT showed low global RVI during the first seconds of ventricular pacing $\left(R V I_{\mathrm{G}}=173.5 \mathrm{~ms}\right.$ dotted line), which further decreased to $R V I_{\mathrm{G}}=110.5 \mathrm{~ms}$ before discontinuing the pacing and the establishment of VT (dashed line). During this interval, the RVI map for this patient showed lower RVI in the apical-inferior LV region, which was therefore associated with higher vulnerability to re-entry (Fig. 3). As documented in a previous study, this region was also associated with undergoing acute ischemia [6].

Both detrending and Bazett's correction compensated for CL dependency of $R V I_{\mathrm{G}}\left(\right.$ Fig 2B-C), while $R V I_{\mathrm{G}}$ esti-
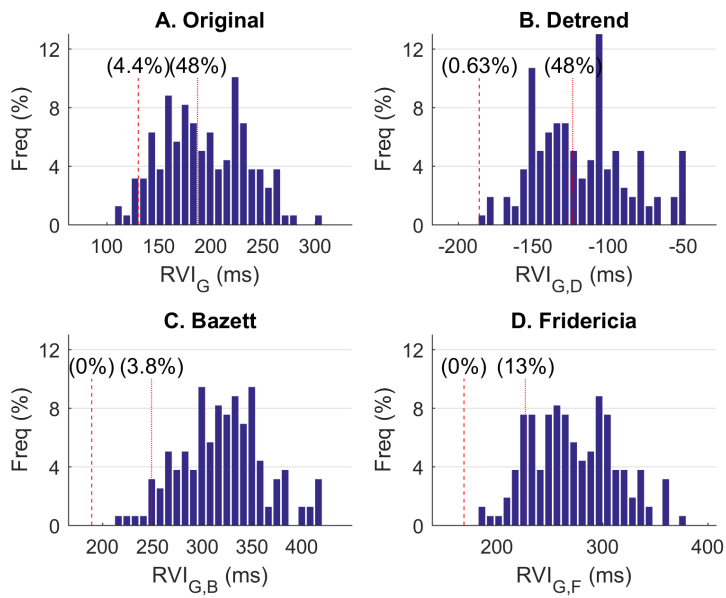

Figure 4. Histograms showing the distribution of global RVI before and after correction, pooling together all patients without VT and CLs. Vertical lines represent global RVI estimated in the VT patient before the onset of VT. The ranking of global RVI estimated in the VT patient with respect to the entire distribution is reported above the line. 
mated after Fredercia's correction still presented a weak positive association with CL (Fig 2D). After corrections for CL dependency, global RVI for the VT patient was still smaller than global RVI for the non-VT patients for all CLs (Fig 2). All corrections enhanced the differences between global RVI measured in the VT patient before the onset of VT and the rest of patients (Fig 4).

\section{Discussion}

This study investigated the CL dependency of RVI, assessed three possible corrections and presented preliminary results about the capability of the global RVI to predict ventricular arrhythmia. The main results are: (1) Own to CL dependency of local repolarization, there was a strong positive association between RVI and CL. (2) Local repolarization detrending and correction with the Bazett's formula eliminated the CL dependency, while a weak association between RVI and CL remained after repolarization correction with the Fredericia's formula. (3) Global RVI calculated before VT onset in the patient that went into VT was significantly lower than global RVI in the 24 patients who did not develop VT. Corrections for CL dependency enhanced these differences. Interestingly, after Bazett's correction, the global RVI calculated during the first seconds of ventricular pacing in the patient that went into VT was already lower than global RVI in the rest of patients, suggesting that global RVI could provide an early assessment of the predisposition to ventricular arrhythmia. The underlying hypothesis of this study is that RVI could be used as a risk stratification marker. The RVI has been designed to capture the interplay between premature activation, conduction slowing and repolarization time that is critical for the establishment of re-entrant tachycardia $[1,10]$. It is function of both repolarization and conduction dynamics and partially correlates with ARI, RT and local dispersion of AT. To compare global RVI among patients with different heart rates, a correction to compensate for the CL dependency of repolarization is necessary. The results of this study suggest that detrending and Bazett's correction eliminate CL dependency in the range from $350 \leq C L \leq 600 \mathrm{~ms}$. However, these results may not apply for different CLs and in particular for $C L<350$ ms, when both dispersion of AT and RT show CL dependency making the interaction more complex [3]. As only one patient who developed VT was included in the study, the results about the predictive value of global RVI should be considered as preliminary and further analysis is necessary to establish the role of RVI as a stratification marker.

\section{References}

[1] Child N, Bishop MJ, Hanson B, Coronel R, Opthof T, Boukens BJ, Walton RD, Efimov IR, Bostock J, Hill Y, Rinaldi CA, Razavi R, Gill J, Taggart P. An activation- repolarization time metric to predict localized regions of high susceptibility to reentry. Heart rhythm 2015; 12(7):1644-53.

[2] Hill YR, Child N, Hanson B, Wallman M, Coronel R, Plank G, Rinaldi CA, Gill J, Smith NP, Taggart P, Bishop MJ. Investigating a Novel Activation-Repolarisation Time Metric to Predict Localised Vulnerability to Reentry Using Computational Modelling. PLoS ONE 2016;11(3):e0149342.

[3] Orini M, Taggart P, Srinivasan N, Hayward M, Lambiase PD. Interactions between activation and repolarization restitution properties in the intact human heart: In-vivo whole-heart data and mathematical description. PLoS ONE 2016;11(9):e0161765.

[4] Srinivasan NT, Orini M, Simon RB, Providência R, Khan F, Segal OR, Babu G, Bradley R, Rowland E, Ahsan S, Chow AW, Lowe M, Taggart P, Lambiase P. Ventricular Stimulus Site Influences Dynamic Dispersion of Repolarization In The Intact Human Heart. American Journal of Physiology Heart and Circulatory Physiology 2016;ajpheart.00159.2016.

[5] Taggart P, Orini M, Hanson B, Hayward M, Clayton R, Dobrzynski H, Yanni J, Boyett M, Lambiase PDP. Developing a novel comprehensive framework for the investigation of cellular and whole heart electrophysiology in the in situ human heart: Historical perspectives, current progress and future prospects. Progress in Biophysics and Molecular Biology 2014;115(2-3):252-260.

[6] Orini M, Taggart P, Hayward M, Lambiase PD. Spatiotemporal characterization of the transition from sinus rhythm to ventricular fibrillation during an acute ischemic event in the intact human heart by whole-heart sock-mapping. HeartRhythm Case Reports 2017;3(5):253-257.

[7] Orini M, Hanson B, Monasterio V, Martínez JP, Hayward M, Taggart P, Lambiase P. Comparative evaluation of methodologies for T-wave alternans mapping in electrograms. IEEE Trans Biomed Eng 2014;61(2):308-316.

[8] Orini M, Citi L, Hanson BMB, Taggart P, Lambiase PDP. Characterization of the Causal Interactions Between Depolarization and Repolarization Temporal Changes in Unipolar Electrograms. In Computers in Cardiology, volume 40. IEEE, 2013; 719-722.

[9] Coronel R, de Bakker JMT, Wilms-Schopman FJG, Opthof T, Linnenbank AC, Belterman CN, Janse MJ. Monophasic action potentials and activation recovery intervals as measures of ventricular action potential duration: Experimental evidence to resolve some controversies. Heart Rhythm 2006;3(9).

[10] Coronel R, Wilms-Schopman FJ, Opthof T, Janse MJ. Dispersion of repolarization and arrhythmogenesis. Heart Rhythm 2009;6(4):537-543.

Address for correspondence:

Name: Michele Orini

Full postal address: University College London, Gower Street, London, UK.

E-mail address: m.orini@ucl.ac.uk 